\title{
TREATMENT OF ADULT PATIENTS WITH CRANIOCEREBRAL TRAUMA COMPLICATED BY VENITALORY FAILURE
}

\section{Jolanta Piskorz ${ }^{1,2}$, Joanna Iłżecka², Gustaw Wójcik ${ }^{3,4}$}

\author{
${ }^{1}$ Department of Anesthesiology and Intensive Care, \\ The Zofia z Zamoyskich Tarnowska Regional Hospital in Tarnobrzeg \\ ${ }^{2}$ Laboratory of Neurological Rehabilitation, Department of Rehabilitation, \\ Physiotherapy and Balneotherapy Medical University of Lublin \\ ${ }^{3}$ Balneotherapy Unit, Department of Rehabilitation, \\ Physiotherapy and Balneotherapy, Medical University of Lublin \\ ${ }^{4}$ Department of Diagnostic Imaging, \\ The Zofia Zamoyski Tarnowska Provincial Hospital in Tarnobrzeg
}

Piskorz J., Iłżecka J., Wójcik G, (2014) Treatment of adult patients with craniocerebral trauma complicated by venitalory failure. Health Problems of Civilization 2 (8), p. 4-9

Summary: This article contains the current state of knowledge on modern methods of treating patients with severe cranio-cerebral injuries, hospitalized in intensive care due to respiratory failure. Severe traumatic brain injury (TBI) can be divided into primary and secondary. Primary brain damage takes place in mechanism of direct injury most often due to an impact. Secondary damage is the result of subsequent disorders of physiological conditions such as ischemia and hypoxia of the brain areas covered by the primary injury. Treatment of patients with severe cranio-cerebral injuries is focused on maintaining proper ventilation, hemodynamic stability and optimal values of intracranial pressure. Maintaining adequate blood flow through brain vessels is necessary to prevent the development of ischemic changes. Respiratory failure develops in these patients by direct injury to the brain in the form of tachypnea, bradypnoea, shortness of breath, sleep apnea, or secondarily to trauma as pneumonia, acute respiratory distress syndrome (ARDS), or pulmonary edema. Mechanical ventilation in patients with TBI seeks to maintain an oxygen partial pressure in the arterial blood of more than $11 \mathrm{kPa}$ and the partial pressure of carbon dioxide in the range of 4.5 to $5.0 \mathrm{kPa}$. The use of appropriate therapy is to prevent the secondary damage to the brain or reduce complications associated with secondary damage.

Key words: cranio-cerebral trauma, respiratory failure, intracranial pressure

\section{Introduction}

Head injuries are the main cause of morbidity and mortality in all age groups. The most common causes. The most common causes of brain injury are motor vehicle accidents, falls, sports injuries, collisions, and assault and violence. Clinical reviews of the European Union show that $40 \%$ of traumatic brain injury was the result of traffic accidents (Tagliaferri et al. 2006).

Craniocerebral trauma is accompanied by neuropathological changes that depend on the type and severity of the injury. Neuropathological changes are divided into focal damage such as bruising of brain tissue, hemorrhage, skull fractures and extensive damage, which include diffuse axonal injury, diffuse vascular damage, ischemia and cerebral edema.

The result of brain contusions are intracerebral hematomas, often accompanied by fractures in the area of contusion. This type of damage is usually the result of trauma contrecoup injury of brain and relate to the frontal lobes, orbital surface of the frontal lobes, orbital surface of the frontal lobes, temporal lobes and side surfaces. Severe brain contusions lead to hemorrhage into the brain parenchyma and increased intracranial pressure.

Another consequence of brain injury is epidural hematoma. It arises in patients with severe head injury, usually accompanied by fracture of the temporal bone husks and tear the middle meningeal artery. The volume of hematomas than $150 \mathrm{ml}$ is a poor prognostic factor (Stienen M et al. 2013).

Address for correspondence: Jolanta Piskorz, Medical University of Lublin, Al. Racławickie 1, 20-059 Lublin, e-mail: jolapis@op.pl phone 506-113-385

Tables: 0, Figures: 0, References: 30, Full text PDF www.hpc.edu.pl Copyright (C) Pope John Paul II State School of Higher Education In Biała Podlaska, Sidorska 95/97, 21-500 Biała Podlaska Indexation: Index Copernicus, Polish Ministry of Science and Higher Education. This is an open-access article distributed under the terms of the Creative Commons Attribution Non-commercial License (http://creativecommons.org/licenses/by-nc/3.0), which permits use, distribution, and reproduction in any medium, provided the original work is properly cited, the use is non-commercial and is otherwise in compliance with the license. 
Subdural hematoma is caused by the rupture of connecting veins, especially those in the vicinity of the upper bay fibula. Most often it is the result of rapid acceleration or inhibition of the head. Subdural hematomas are divided into acute when formed shortly after the injury, sub-acute appearing 1-2 weeks after injury, and chronic when there are more than two weeks after the injury. Patients of Intensive Care Unit are usually hospitalized with acute subdural haematomas after surgical decompression of the hematoma.

Often, brain contusions and rupture are accompanied by accumulation of blood in the sub-arachnoid space. Massive sub-arachnoid hemorrhage can occur due to rupture of the vertebral artery, basilar artery of the brain or another. This event is often fatal (Geraldes et al. 2014).

Cerebral edema occurs commonly in patients with cranio-cerebral injuries. There is a vasogenic edema, brain injury as a result of cerebral vasodilation in which there is an increase in blood volume flowing through the brain or to leakage of cerebrospinal fluid as a result of failure of the blood-brain barrier. Cytotoxic edema occurs when there is an increase in intracellular water content of the central nervous system. Cerebral edema is the cause of raised intracranial pressure and cerebral blood flow pressure drop, leading to ischemic brain damage.

Trauma, hypoxia, ischaemia and hypoglycaemia, often lead to the destruction of axons within the brain. Patients with diffuse traumatic axonal are unconscious, and the prognosis is poor. Most end up fatal or serious injury or permanent vegetative state (Sullivan et al. 2013).

\section{Selected treatment of patients with cranio-brain injuries}

Intensive treatment of patients with severe brain injuries includes elements of surgical and conservative treatment. Cerebral ischemia is the most important secondary factor that influences the outcome. To maintain the normal range of brain perfusion pressure, the correct mean arterial pressure must be provided. It is indicated for hemodynamic stability and avoidance of hypotension through proper filling of the vascular bed. Isotonic crystalloid and colloid are applied, and if necessary, blood decongestants (Johnston et al. 2004).

In post-traumatic intracranial pressure monitoring (ICP) appropriate sedation, analgesia and muscle relaxants as needed play a crucial role. Most of sedative act as an anticonvulsant. Muscle relaxants affect the optimization of the mechanical ventilation in patients with severe traumatic brain injuries, minimize coughing and muscle tone which are responsible for, inter alia, the increase of ICP. The most common drugs used for sedation is propofol and short acting benzodiazepine - midazolam. They cause a dose-dependent reduction of cerebral oxygen metabolism and cerebral blood flow (Johnston et al. 2003). Propofol acts quickly and briefly, therefore, allows for rapid assessment of the neurological status of the patient. It is given as a continuous infusion. It has a beneficial effect on the intracranial homeostasis, does not interfere with the mechanism of autoregulation of cerebral circulation and cerebral vascular reactivity in response to the change of carbon dioxide (Tobias 2000). While using of propofol, there may, however, occur a cardio-respiratory collapse and the propofol treatment syndrome (Kumar et al. 2005).

In the treatment of patients with brain injuries, a very important aspect is pain therapy. Suitable doses of paracetamol, and opioids provide analgesia to such extent to avoid increased ICP. The most commonly used are: morphine, fentanyl and remifentanil. Fentanyl and remifentanil have short duration but a continuous infusion of fentanyl results in accumulation in tissues and remifentanil has a half-life of less than 5 min, therefore, does not accumulate.

Barbiturates are another group of agents used in the treatment of severe cranio-cerebral. They reduce ICP, decrease the metabolism of the brain, impair vascular hemodynamics, reduce cellular acidosis (Mansour et al. 2013). The infusion of barbiturates, especially in large doses is a cause of hypotension due to impaired venous return, baroreceptor reflex inhibition and a depressive effect on the heart muscle, therefore their use should be cautious and justified by refractory intracranial pressure that does not respond to any other treatment.

Patients with severe brain injuries require respiratory support with mechanical ventilation. Mechanical ventilation should be included in the early stages of cranio-cerebral treatment to avoid hypoxemia and hypercapnia, which secondarily leads to the development of intracranial hypertension (Ghajar, 2000). One have to choose the kind of mechanical ventilation to maintain oxygen tension and carbon dioxide in the arterial blood within the normal range. Patients after neurosurgical and trauma surgeries of the skull or brain tumors usually have increased ICP. Using ventilation with positive pressures, especially PEEP (positive pressure ventilation with end-tidal) decreases venous return from the head which leads to an increase in ICP. Another mechanism that leads to disruption of cerebral blood flow during ventilation with PEEP is a decrease in cardiac output and, consequently, a decrease in mean arterial pressure and cerebral perfusion pressure (Caricato et al. 2005). When the value of the ICP is high, ventilation with PEEP should be carefully used. Helmy et al. reported that PEEP to $12 \mathrm{~cm} \mathrm{H} 20$ does not cause a dangerous increase in ICP (Helmy et al. 2007). 
In clinical practice, in order to reduce ICP hyperventilation applies also to bring about a drop in the pressure of carbon dioxide in arterial blood. Lowering PaCO2 causes cerebral vasoconstriction and parallel decrease in cerebral blood flow and the ICP. This practice by is not recommended by many authors, because vasoconstriction due to hypocapnia may worsen cerebral ischemia and cause cerebral lactic acidosis. (Solaiman, Singh 2013). In addition, if you are trying to restore the value of $\mathrm{PaCO} 2$ by reducing hyperventilation, there may be an increase in ICP with reflection, which results in excessive blood supply to the brain tissue as a result of reperfusion injury and in places previously ischemic bleeding may occur (Marik et al. 2002).

In the treatment of cranio-cerebral trauma, in order to reduce the ICP osmotic diuretics and concentrated solutions of sodium chloride are applied. Mannitol is currently only used osmotic diuretic. After intravenous use no backflow is absorbed in the renal tubules, depending on the osmotic pressure, the water is stopped in the light of the channel and the diuresis is increased. With the intact blood-brain barrier, mannitol creates the osmotic gradient and the displacement of water from the vascular compartment reduces brain swelling. At osmolarity more than 320 mOsm / l supply of this drug is contraindicated because it increases neurological and renal side effects (Ye, Su 2013).

Hypertonic sodium chloride solution has an osmotic effect by displacing the water in accordance with the osmotic gradient from the brain parenchyma into the intravascular space, so the tissue pressure is decreased, cell size, and thereby, the swelling of the brain is reduced (Diringer 2013).

Patients with cranio-cerebral injuries often suffer from cardio-respiratory and systemic complications. The cause of them is direct brain injury and concomitant injuries and complications of treatment. Zyguan et al argue that the failure of one organ is associated with a mortality rate of about $40 \%$, in case of failure of two organs increases to about $47 \%$, and if the damage is three or more bodies mortality reaches 100\% (Zyguan et al. 2005).

Cardiovascular complications after brain injuries occur in the form of hemodynamic instability, relative hypotension, which requires the use of vasoactive agents in the treatment and in the form of disturbances in heart rhythm, often requiring urgent intervention (supraventricular tachycardia, ventricular fibrillation). Acute cardiac failure while brain injury is probably secondary to the accompanying increase in the concentration of catecholamines [Macmillan et al. 2002]. The commonly used drugs are vasoactive epinephrine, norepinephrine, dopamine and dobutamine. Sympathomimetics are working on the principle of stimulation of naturally occurring adrenergic receptors. They are used to increase the mean arterial blood pressure and cerebral perfusion pressure, as well as in patients who developed neurogenic pulmonary edema, myocardial dysfunction and organ failure. Before using vasoactive drugs, one should always rule out hypovolemia and make up the volume of circulating blood. Recommendations of Brain Trauma Foundation in 2007 are such that brain perfusion pressure ought be maintained at 50-70 mmHg but aggressive maintaining this pressure above $70 \mathrm{mmHg}$ by using fluids and vasoactive drugs is not recommended due to the increased incidents of respiratory distress syndrome (The Brain Trauma Foundation, 2007).

Neurogenic pulmonary edema is another consequence of brain injury. The treatment in this case is based on the use of oxygen therapy, ventilator support with respirator, and dobutamine which improves function of left ventricular. Diuretics and nitrates should be used with caution, as they cause a reduction in blood pressure, and decrease in blood and cerebral perfusion pressure (Scalf et al. 2013).

Patients with traumatic brain injury often have respiratory complications in the form of pneumonia, choke and ARDS (Acute respiratory distress syndrome). Pneumonia develops in these patients as a result of different mechanisms, the most common are: aspiration of secretions from the nasopharynx in patients in a coma, elderly age, carrier state of Staphylococcus aureus, the infusion of drugs that cause sedation and barbiturates, which have a immunosuppressive effect. In the treatment of pulmonary inflammation, the most important is early targeted antibiotic therapy, the use of tubes for intubation with the gasketing cuffs of small volume and low pressure, which prevent microaspiration (Young et al. 2006).

ARDS is a severe lung injury where PaO2/FiO2 ratio is less than 200. ARDS is a common complication in patients with brain injuries, associated with a higher risk of death and neurological complications. Therapy involves, inter alia, the use of so-called. saving ventilation, which means the use of small tidal volumes to avoid over-expansion pulmonary barotrauma and decrease in systemic pressure, because the lungs in ARDS are functionally small (Petrucci, Iacovelli 2004).

\section{Modern methods of preventing cranio-cerebral trauma complications}

The result of treatment of patients with craniocerebral trauma is undoubtedly great influenced by primary brain injury severity, but many factors that occur in the post-trauma phase contribute to traumatic secondary brain damage. Secondary damage worsens morbidity and increases mortality. Intracranial hypertension, hypoxemia, hypocapnia, systemic hypotension, fever, hyperglycemia and hypoglycemia are the factors that secondarily damage brain. Suitable treatment of patients with traumatic brain injury is the basis of a neurological intensive care. 
Yan E and et al. on the basis of tests carried out in 44 patients with traumatic brain injury show that in secondary hypoxic brain involves greater production of cytokines and growth levels of biomarkers in serum, which is associated with chronic neurogenic inflammation and correlates with poor prognosis (Yan et al. 2013).

It has long been believed that the cooling of the patients has a neuroprotective effect. Body temperature in the range of 36.50 to $37.50 \mathrm{C} \mathrm{C}$ allows to maintain proper homeostasis. CNS temperature below $350 \mathrm{C}$ is defined as hypothermia. Implementation of hypothermia in the treatment of patients with brain injuries is controversial, but it id used due to the effect of decreasing the ICP (Polderman et al. 2002). Farag E et al at in their work, report that use of hypothermia in patients with traumatic brain injury in secondary trauma treatment is not recommended. In contrast, it is important to maintain normothermia and avoid hyperthermia (Farag et al. 2011).

Secondary brain damage is caused by intracranial hypertension and cerebral edema. One of the methods of treatment of cerebral edema is the use of osmotically active agents. The most used are mannitol and hypertonic sodium chloride solution. Aside from decreasing ICP, they also improve the blood supply to the brain and improve the conditions for the distribution of oxygen There are studies that prove that it seems preferable to use hypertonic sodium chloride solution than mannitol, as in patients with multi-organ injuries contributes to hemodynamic stability and prevent secondary complications. Furthermore, concentrated solutions of salts have the neurohumoral and immunological effect, which may be beneficial in the brain resuscitation (Grape, Ravussin 2012).

Grande and Romner in their work draw attention to the need for osmoteraphy to prevent acute compression of the brain stem until the decompressive procedures are performed, for instance, craniotomy evacuating the hematoma. In contrast, the routine use of a hypertonic solution of mannitol and sodium chloride is not favorable due to a number of complications, inter alia, electrolyte disturbances, renal failure progression, rebound increase in ICP after discontinuation of the infusion of osmotically active factors (Grand, Romner 2012).

If there is damage to the blood-brain barrier, mannitol supply leads to cerebral edema and axonal destruction by the disintegration of myelin sheaths. P. Kozler et al conducted a study which shows that in such cases, used intravenous methylprednisolone can effectively reduce the damage to axons (Kozler et al. 2011).

The development of respiratory complications in patients with traumatic brain injury is quite often. ARDS is a severe lung damage associated with high mortality. There are many methods for the treatment of acute respiratory distress syndrome. One of the methods less commonly chosen due to low availability of such respirators is the high frequency ventilation (HFV). Vrettou et al show advantages in comparison with a conventional mechanical ventilation (CMV). They measured the effect on the pressure of oxygen and carbon dioxide in the arterial blood, systemic hemodynamics, ICP, and the pressure of cerebral perfusion in patients with traumatic brain injury. These researchers concluded that the use of HFV in patients with ARDS with traumatic brain injury improved oxygenation and respiratory mechanics without negative impact on PaCO2, hemodynamics and ICP (Vrettou et al. 2013).

Very important in the treatment of patients with cranio - cerebral injuries is leveling fluid and electrolyte and metabolic disturbances. Levelling of water and electrolyte balance is to maintain homeostasis. Patients should be adequately hydrated to maintain normal plasma volume, left ventricular filling pressure, cardiac output and blood pressure and adequate tissue oxygenation. The purpose of such treatment is to prevent the secondary damage to neurons due to insufficient supply of tissues with oxygen. Clifton et al in their work found that maintaining a negative fluid balance below $-594 \mathrm{ml}$ in a day is associated with a worse prognosis. There is no reliable data indicating justification of the validity limit fluid intake in patients with head injury in order to reduce swelling of the brain (Clifton et al. 2002). Maintaining of normowolemii and normal physiological indicators on cerebral blood flow is essential in the treatment of these patients.

\section{Conclusions}

1 Treatment of patients with severe cranio-cerebral injuries is focused on maintaining normal intracranial pressure, ensuring adequate ventilation and hemodynamic stability.

2 Respiratory failure develops in these patients as a result of direct injury to the brain in the form of tachypnea, bradypnoea, shortness of breath, sleep apnea, or secondary to trauma as pneumonia, ARDS or pulmonary edema. 3 Maintaining normal cerebral blood flow is necessary to prevent the development of ischemic changes. 4 The use of appropriate therapy is to prevent secondary brain damage or reduce the complications associated with secondary damage. 


\section{References:}

1. Caricato A., Conti G., Delia Corte F., Mancino A., Santilli F., Sandroni C. (2005), Effect of PEEP on the intracranial system of patients with head injury and subarachnoid hemorrhage: the role of respiratory system compliance. Trauma. 58(3): s. 571-576.

2. Clifton GL., Miller ER., Coi SC., Levin HS. (2002), Fluid thresholds and outcome from severe brain injury. Crit Care Med. 30(4): s. 739-745.

3. Diringer M.N. (2013), New trends in hyperosmolar therapy. Curr Optin Crit Care. 19(2): s. 77-82.

4. Farag E., Manno EM., Kurz A. (2011), Use of hypothermia for traumatic brain injury: point of view. Minerva Anesthesiol. 77(3): s. 336-370.

5. Geraldes R., Sousa P.R., Fonseca A.C., Falcao F., Canhao P., Pinho E., Melo T. (2014), Nontraumatic convexity subarachnoid hemorrhage: Different etiologies and outcomes. Journal of Stroke and Cerebrovascular Diseases. 23(1): s. 23-30.

6. Ghajar J. (2000), Traumatic brain injury. Lancet. 356: s. 923-929.

7. Grape S., Ravussin P. (2012), PRO: osmotherapy for the treatment of acute intracranial hypertension. J Neurosurg Anesthesiol. 24(4): s.402-406.

8. Grände PO., Romner B. (2012), Osmotherapy in brain edema: a questionable therapy. J Neurosurg Anesthesiol. 24(4): s. 407-412.

9. Helmy A., Vizcaychipi M., Gupta AK. (2007), Traumatic brain injury: intensive care management. Br J Anaesth. 99(1): s. 32-42.

10. Johnston AJ., Steiner LA., Chatfield DA. (2003), Effect of propofol on cerebral oxygenation and methabolism after head injury. Br J Anesth. 91: s. 781-786.

11. Johnston AJ, Steiner LA, Chatfield DA. (2004), Effect of cerebral perfusion pressure augmentation with dopamine and norepinephrine on global and focal brain oxygenation after traumatic brain injury. Itens Care Med. 30: s. 791-797.

12. Kozler P., Riljak V., Pokorný J. (2011), Methylprednisolone reduces axonal impairment in the experimental model of brain oedema. Neuro Endocrinol Lett. 32(6): s. 831-835.

13. Kumar MA., Urrutia VC., Thomas CE., Abou-Khaled KJ. (2005), The syndrome of irreversible acidosis after prolonged propofol infusion. Neurocrit Care. 3: 257-259.

14. Macmillan CS., Grant IS., Andrews PJ. (2002), Pulmonary and cardiac sequelae of subarachnoid haemorrhage: time for active management? Intens Care Med. 28: s. 1012-1023.

15. Mansour N., deSouza R.M., Sikorski C., Kahana M., Frim D. (2013), Role of barbiturane coma In the management of focally induced, severe cerebral edema In children. 12(1): s. 37-43.

16. Marik PE., Varon J., Trask P. (2002), Management of head trauma. Chest. 122(2): s. 699-711.

17. Petrucci N., Iacovelli W. (2004), Ventilation with lower tidal volumes versus traditional tidal volumes $n$ adults for acute lung injury and acute respiratory distress syndrome. Cochrane Database Syst Rev. (2): s. 384-396.

18. Polderman KH., Tjong Tjinoe R., Peerdeman SM., Vandertrop WP., Girbes AR. (2002), Effect of therapeutic hypothermia on intracranial pressure and outcome in patients with severe head injury. Intens Care Med. 28: s.15631567.

19. Scalfani M.T., Dhar R., Zazulia A.R., Videen T.O., Diringer M.N. (2013), Effect of osmotic agents on regional cerebral blood flow in traumatic brain injury. J Crit Care. 27(5): s. 7-12.

20. Solaiman O., Singh J.M. (2013), Hypocapnia in aneurismal subarachnoid hemorrhage: Incidence and association with poor clinical outcomes. Journal of Neurosurgical Anesthesiology. 25(3): s. 254-261.

21. Stienen M., Abdulazim A., Hildebrandt G., Gautshi 0.(2013), Emergency scenario: epidural hematoma- evalution and management. Praxis (Bern). 30: s.102-103.

22. Sullivan G.M., Mierzwa A.J., Kijpaisalratana N., Tang H., wang Y., Song S.K., Selwyn R., Armstrong R.C. (2013), Oligodendrocyte lineage and subventricular zone response to traumatic axonal injury in the corpus callosum. J Neuropathol Exp Neurol. 72(12): s.1106-1125.

23. Tagliaferri F., Compagnone C., Korsic M. (2006), A systematic review of brain injury epidemiology in Europe. Acta Neurochir (Wien). 148: s. 255-268.

24. The Brain trauma Foundation and the Joint section of the American Association of Neurological Surgeons and Congress of Neurological Surgeons on Neurotrauma and Critical Care. (2007), Guidelines for the Management of Severe Traumatic Brain Injury. $3^{\text {rd }}$ edition. IX Cerebral Perfusion Thresholds. J Neurotrauma. 24: s. 59-64.

25. Tobias JD. (2000), Propofol: effect on the central nervous system. J. Intensive Care Med. 15(5): s. 237-246. 
26. Vrettou CS., Zakynthinos SG., Malachias S., Mentzelopoulos SD. (2013), High-frequency oscillation and tracheal gas insufflations in patients with severe acute respiratory distress syndrome adn traumatic brain injury: an interventional physiological study. Crit Care. 17(4): s. 136-140.

27. Yan E., Satgunaseelan L., Paul E., Bye N., Nyguyen P., Agyapomaa D., Kossmann T., Rosenfeld JV., Morganti-Kossmann C. (2013), Post-traumatic hypoxia is associated with prolonged cerebral cytokine production, higher serum biomarker levels and poor outcome in patients with severe traumatic brain injury. J Neurotrauma. 11(26): s. 1146-1160.

28. Ye H., Su Y. (2013), Hemodynamic effect of mannitol infusion in patients with acute intracerebral hemorrhage. Acta Cir Bras. 28(2): s. 106-111.

29. Ypung PJ., Pakeerathan S., Blunt MC., Subramanya S. (2006), A low-volume, low-pressure tracheal tube cuff reduces pulmonary aspiration. Crit Care Med. 34: s. 632-639.

30. Zyguan DA., Kortbeek JB., Fick GH., Laupland KB., Doig CJ. (2005), Non-neurologic organ dysfunction in severe traumatic brain injury. Crit Care Med. 33: s. 654-660.

Submitted: 28.04.2014

Accepted: 06.06.2014 